Pathologe 2018 · 39:415-423

https://doi.org/10.1007/s00292-018-0474-2

Online publiziert: 22. August 2018

(c) Der/die Autor(en) 2018

\section{Schwerpunktherausgeber}

W. Saeger, Hamburg

K.W. Schmid, Essen

CrossMark

\title{
G. Mikuz
}

Institut für Pathologie, Universität Innsbruck, Innsbruck, Österreich

\section{Ektopien der Niere, Harnwege und männlichen Geschlechtsorgane}

\section{Ektopie der Niere}

genital Anomalies (EUROCAT) waren im Zeitraum 1996-2001 angeborene Anomalien des Urogenitaltrakts (mit Ausnahme des äußeren Genitale) mit 28/10.000 Lebendgeborenen am dritthäufigsten nach kardiovaskulären Anomalien (61/10.000 Lebendgeborenen) und Anomalien der Glieder (37/10.000 Lebendgeborenen; [21]). Die häufigsten Fehlbildungen des Urogenitaltraktes betreffen die Niere, wobei die Fehllagerungen sicherlich an erste Stelle stehen. Ektopien der übrigen Urogenitalorgane sind hingegen, mit Ausnahme der Ureteren, extrem selten.

Pathologen sehen Ektopien nur im Seziersaal und nicht in der täglichen histologischen Diagnostik. Dementsprechend werden selbst in Uropathologiebüchern diese Fehllagerungen, wenn überhaupt, nur sehr kurz besprochen. Insgesamt beruht unser diesbezügliches Wissen auf unzähligen Kasuistiken, die in den 1960er- und 1970er-Jahren von Urologen und Kinderärzten publiziert wurden. Durch die moderne medizinische Bildgebung werden Ektopien häufiger zufällig „sichtbar“, deswegen findet man neuerdings die meisten Publikationen in Zeitschriften der Radiologie. Eine echte „Forschung“ auf diesem Gebiet hat es, mit wenigen Ausnahmen [27], nicht gegeben.

Die englische Version dieses Beitrags ist unter https://doi.org/10.1007/s00292-018-0505-z zu finden.
Die echte Ektopie der Niere sollte von der Nephroptose (Nierenptose, Senk- oder Wanderniere) unterschieden werden. In diesem Fall nimmt das Organ im Liegen die normale Position an, wandert aber im Stehen etwa $5 \mathrm{~cm}$ kaudalwärts.

\section{Embryologie}

In der 5. Schwangerschaftswoche entsteht die endgültige Niere aus dem metanephrogenen Blastem und der Ureterknospe. Letztere ist eine Ausstülpung des Wolff-Ganges (Ductus mesonephricus), aus dem sich Ureter, Nebenhoden, Ductus deferens, Samenblase und Ductus ejaculatorius entwickeln [19].

Der Metanephros liegt ursprünglich auf Höhe der Bifurkation der Aorta. Zwischen der 6. und 9. Woche beginnt der sog. Aszensus, der Aufstieg zur endgültigen Lage in der Höhe des 12. Brustwirbels. Beim Erreichen der endgültige Lage in der Fossa lumbalis kommt es zur Rotation der Niere mit Ausrichtung des Nierenbeckens nach medial und der Kelche nach lateral.

Das Ausbleiben des Aszensus ist die Ursache der Nierenektopie im Beckenbereich, wobei die genaue Ursache dafür praktisch unbekannt ist. Als mögliche Ursachen werden eine mangelnde Ausbildung der Ureterknospe, krankhaftes metanephrogenes Blastem, Anomalien der Arterien, die die Nieren zu verschiedenen Entwicklungsstufen versorgen, sowie unbekannte genetische Störungen vermutet [19].

Die Ektopie der Niere im thorakalen oder subdiaphragmalen Raum entsteht hingegen durch einen schnelleren Aszensus. Dadurch erreicht das Organ die normale Lage, noch bevor sich das Zwerchfell verschließt (8. Schwangerschaftswoche). Die andere Möglichkeit ist, dass der Diaphragmaverschluss gestört und/oder verlangsamt ist [28].

Gleichzeitig mit der Ektopie findet oft auch eine Malrotation statt. Das Nierenbecken ist anstatt nach medial nach ventral, dorsal oder lateral ausgerichtet [34]. Die Malrotation kann selten auch als alleinige renale Missbildung vorkommen.

Genetisch bedingt sind nur Nierenektopien, die im Rahmen eines komplexen Missbildungssyndrom auftreten.

\section{Morphologie}

Die ektope Niere kann überall im Retroperitoneum vorkommen. Mit der einfachen oder ungekreuzten renalen Ektopie bezeichnet man eine Niere, die zwar auf der richtigen Seite, aber nicht in der Fossa lumbalis liegt. Bei der gekreuzten renalen Ektopie [25] liegt die Niere auf der gegenüberliegenden Seite, was häufig zur Fusion beider Nieren führt. Daher unterscheidet man noch die gekreuzte Ektopie mit oder ohne Fusion beider Nieren. Extrem selten wurden Nieren auch intrathorakal und subdiaphragmal beobachtet.

Bei der einfachen (ungekreuzten) Nierenektopie liegt eine Niere im kleinen Becken in der Fossa iliaca (lumbale/abdominale Niere) oder unterhalb der Aortenbifurkation (Beckenniere) bei gleichzeitiger normaler Lage der kontralateralen Niere ( $\bullet$ Abb. 1a). Das ektope Organ ist nach vorn rotiert und hat meist eine abnorme Form. 

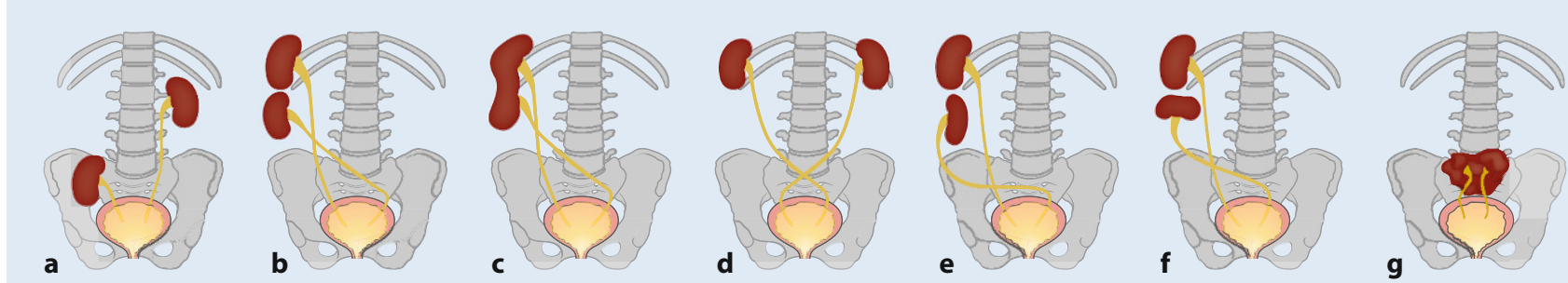

Abb. $1 \Delta$ a Einfache Nierenektopie. b-g Gekreuzte Nierenektopien: b lange Niere, c fusionierte lange Niere, d bilateral gekreuzte Nierenektopie, e Sigmaniere, $\mathbf{f}$ L-Niere, $\mathbf{g}$ Kuchenniere

Bei der gekreuzten Ektopie liegt die Niere auf der kontralateralen Seite in Bezug zur Uretermündung in die Harnblase, d.h., ihr Ureter kreuzt die Mittellinie des Körpers. Nach der Klassifikation von McDonald und McClellan [25] werden folgende Varianten beobachtet:

- Lange Niere (häufigste Variante)

- die Nieren stehen übereinander

( $A$ Abb. 1b) und sind oft fusioniert

(- Abb. 1c).

- Bilateral gekreuzte Nierenektopie (seltenste Variante) - die Nieren liegen in der Fossa lumbalis, beide Ureteren sind gekreuzt und liegen jeweils auf der kontralateralen Seite

(• Abb. 1d).

- Sigmaniere (zweithäufigste Variante) - die Nieren stehen übereinander. Das Nierenbecken der orthotopen Niere ist normal in Richtung median gerichtet, das der gekreuzten zeigt hingegen auf die gekreuzte Seite, d.h., es ist nach außen gerichtet (- Abb. 1e). Die Lage erinnert ein wenig an den griechischen Buchstaben Sigma $(\Sigma)$.

- L-Niere - die gekreuzte Niere liegt horizontal unterhalb der orthotopen, was wie der Großbuchstabe L anzusehen ist (• Abb. 1f).

- Solitäre gekreuzte Ektopie - es fehlt die ungekreuzte Niere.

Warum die Kuchenniere in allen diesbezüglichen Publikationen zu den fusionierten gekreuzten Ektopien gezählt wird, obwohl die Ureteren nicht die Mittellinie kreuzen und orthotop münden, ist eigentlich nicht verständlich. Bei der Kuchenniere (engl. „pancake kidney“, „discoid kidney“, „disc kidney“, „lump kidney“, „fused pelvic kidney“ oder „cake kidney“) sind sowohl der untere als auch der obere Pol zusammengewachsen, womit eine eher unförmige kuchenähnliche Masse entsteht, die vor der Aortenbifurkation liegt. Die Nieren haben auch nicht rotiert, deswegen liegen die beiden Ureteren parallel an der ventralen Seite der beiden getrennten Nierenbecken und münden ungekreuzt in der Blase $(\mathbb{A} \mathbf{b} b \mathbf{b} . \mathbf{1 g})$. Ein solitärer Ureter ist selten.

Die gelegentlich erwähnte Scheibenniere ist eine Variante der Kuchenniere, bei der die Form beider fusionierten Organe einigermaßen erhalten ist und auch die gegenüberliegenden Nierenbecken gut erkennbar sind. In der oben angeführte angelsächsische Nomenklatur werden „disc kidney“ bzw. „discoid kidney“ oft als Synonym für Kuchenniere verwendet.

\section{Klinik}

Die Häufigkeit der einfachen Nierenektopie wird in der Literatur sehr unterschiedlich angegeben. In alten autoptischen Untersuchungen wird die Häufigkeit mit 1:900 [1], in radiologischen Arbeiten mit 1:3000 Untersuchungen angegeben [5]. Eine solitäre Nierenektopie, d.h. ektope Niere mit kontralateraler Nierenagenesie, ist mit 1:22.000 extrem selten und hat meist eine Beckenlage [10]. Frauen und Männer sind gleich häufig betroffen. Bei Frauen wird die abnorme Lage aber häufiger klinisch diagnostiziert, weil sie häufiger an Harninfekten leiden, die nicht mit der Ektopie in Zusammenhang stehen. Patienten mit einfacher Nierenektopie zeigen keine vermehrte Neigung zu Harnstauungen und/oder Niereninfekten [11], d.h., das Leiden wird zufällig bei radiologischen Untersuchungen oder im Seziersaal diagnostiziert.
Die Inzidenz der gekreuzten renalen Ektopie wird von Radiologen mit 1:7000 Patienten geschätzt [5]. In bis $90 \%$ der Fälle kommt es zur einen Fusion der orthotopen mit der ektopen Niere [25], die in der Regel, mit extrem seltenen Ausnahmen, unterhalb der orthotopen liegt. Darüber hinaus leiden Betroffene nicht selten an anderen Missbildungen der Harnwege (Megaureter, Ureterektopie, ureterale Klappen, vesikoureteraler Reflux; [18]) oder des Skeletts $[18,29]$. Die gekreuzte renale Ektopie ist auch Teil des akrorenalen Syndroms (AROS) das mit Augenanomalien (Kolobom des N. opticus), Missbildungen der Handknochen (Knochenfusion der Phalangen, Polydaktylie, Oligodaktylie usw.) und der Harnwege (Nierenektopie, Malrotation, beidseitige Nierenhypoplasie, Hufeisenniere, Blasendivertikel) einhergeht [29].

Ähnlich der einfachen Ektopie verläuft auch die gekreuzte meist symptomlos, kann aber insbesondere bei Kindern zum Harnreflux oder -stau führen, und benötigt somit eine chirurgische Korrektur.

Die subdiaphragmalen und intrathorakalen Nierenektopien sind mit 1:22.000 Patienten sehr seltene Ereignisse ([5]; Abb. 2). Subdiaphragmal gelegene Nieren findet man nicht selten in Patienten mit Omphalozele $[2,30]$. Weil die Leber nicht subdiaphragmal, sondern im Omphalozelensack liegt, kann wahrscheinlich die Niere ungehindert höher aufsteigen [30].

Man vermutet, dass die nicht subdiaphragmal, sondern im Omphalozelensack liegende Leber den Nierenaszensus nicht stoppen kann [30].

Wie bereits erwähnt entsteht die „echte“ intrathorakale Ektopie wahr- 
scheinlich durch einen beschleunigten Aszensus. Andere Ursachen sind die Eventration $^{1}$ des Zwerchfells, die Hiatushernie und traumatische Rupturen [9]. Selbst bei dieser extremen, oft zufällig entdeckten Fehllagerung kann das Organ ganz normal funktionieren.

\section{Ektopie des Ureters}

Die normal verlaufenden Harnleiter münden am Blasengrund im Trigonum vesicae. Jede Mündung außerhalb dieses Bereichs muss als Ektopie bezeichnet werden.

\section{Embryologie}

Der Ureter entsteht aus der Ureterknospe, einer Ausstülpung des Wolff-Ganges, die nach Aufwärtswanderung (Migration) und Rotation in die Wand der Harnblase einverleibt wird und im Bereich des Trigonums mündet. Wenn diese Ausstülpung zu hoch kranialwärts angesiedelt ist, sind Rotation und Absorption in den urogenitalen Sinus verzögert, d.h., die Uretermündung hat keine Zeit um das Trigonum der Blase zu erreichen und inseriert entweder im kranialen Teil der Urethra oder beim Mann in der prostatischen Urethra.

Wenn die Ureterknospe sehr hoch liegt, kommt es nicht zur Trennung vom Wolff-Gang, der Ureter drainiert deswegen in ein Organ des Genitaltraktes. Beim Mann sind dies die Abkömmlinge des Wolff-Ganges. Bei der Frau verschwindet der kraniale Teil dieses Ganges, Teile davon werden im MüllerGang einverleibt oder bilden als Relikt den Gartner-Gang, der auf beiden Seiten des Uterus und der Vagina im Ligamentum latum uteri bzw. in der Vaginalwand liegt. Somit erklärt sich die häufige Uretermündung in Vulva und Vagina.

\section{Morphologie}

Wie aus der Organogenese der Harnleiter ersichtlich ist, kann deren Orificium sowohl in Blasenhals und Urethra als auch

\footnotetext{
1 Abnorme Ausbuchtung wegen angeborener
} Schwäche derZwerchfellmuskulatur

Pathologe 2018 $\cdot 39: 415-423$ https://doi.org/10.1007/s00292-018-0474-2

(c) Der/die Autor(en) 2018

\section{G. Mikuz}

\section{Ektopien der Niere, Harnwege und männlichen Geschlechtsorgane}

\section{Zusammenfassung}

Eine der häufigsten Missbildungen der Harnwege ist die einfache Ektopie der Niere, bei der das Organ im kleinen Becken liegt. Bei der etwas selteneren gekreuzten Ektopie liegt die Niere auf der kontralateralen Seite in Bezug zur Uretermündung in die Harnblase, d.h., ihr Ureter kreuzt die Mittellinie des Körpers. Die Ursache dafür ist das Ausbleiben des Aszensus, des Aufstiegs der ursprünglichen Niere aus dem Becken in die orthotope Lage. Ein beschleunigter Aszensus führt hingegen zur intrathorakalen Nierenektopie. Ektope Ureteren münden außerhalb des Trigonum vesicae. Bei Störungen in der Organogenese kann deren Orificium sowohl in Blasenhals und Urethra als auch im Bereich der Geschlechtsorgane liegen.

Die Blasenekstrophie ist ein fehlender Verschluss der vorderen Bauchwand, sodass die Blasenschleimhaut in die umgebende Haut übergeht. Bei der kloakalen Ekstrophie ist die Harnblase gespalten, dazwischen liegt der ileozökale Anteil des Darms. Als
Hodenektopie bezeichnet man die Lage des Organs außerhalb der Deszensuswege. Bei der Ektopie der Prostata handelt sich nicht um eine Fehllagerung des gesamten Organs, sondern um versprengte Prostatadrüsen, die vor allem in der Blasenwand und in der Urethra zu finden sind. Einige Fälle wurden aber auch in der Darmwand, im Anus, im perikolischen Fettgewebe, Milz, Samenblase, Hoden sowie in der Cervix uteri und Vagina beschrieben.

Die Penis- und Skrotumektopie und die penoskrotale Transposition sind die seltensten Missbildungen der männlichen Geschlechtsorgane überhaupt. Die seltenste Form ist die gemeinsame Verlagerung von Penis und Skrotum ins Perineum. Bei der Transposition liegt hingegen der Penis unter dem Skrotum oder zwischen beiden Hoden in einer Furche des gespaltenen Hodensacks.

Schlüsselwörter

Blasenekstrophie - Choristom - Embryologie . Kloake $\cdot$ Penoskrotale Transposition

\section{Ectopias of the kidney, urinary tract organs, and male genitalia. German version.}

\section{Abstract}

The most frequent anomaly of the urogenital tract is a simple renal ectopia with one organ lying in the pelvis. Crossed renal ectopia is a less common condition in which the ectopic kidney is located on the opposite side of the midline from the ureteral insertion in the urinary bladder. The cause of both types of renal ectopia is the arrest or failure of the kidney ascent from the pelvic to the lumbar position. Whereas an accelerated ascent leads to a subdiaphragmal or intrathoracic ectopic position, an ectopic ureter can be defined as one that does not drain into the trigonum vesicae. The ectopic orificium can be located situated in the bladder neck and urethra as well as somewhere in the genital area.

Exstrophy of the urinary bladder is not a complete ectopia. Because the abdominal wall and the anterior part of the bladder wall are lacking, the bladder mucosa grows directly into the skin. The complex exstrophy of the bladder and intestine corresponds to a cloacal exstrophy, in which the bladder is split in two halves on either side of the gut portion. Testicular ectopia refers to the location of the testis in a position outside of its normal course of descent.

Prostatic ectopia does not refer to the wrong location of the entire organ, but to a scattered group of prostate glands, which are mostly found in the submucosal part of the urinary bladder or proximal urethra. Other described locations are the intestinal wall, anus, pericolic fat tissue, spleen, seminal vesicle, testis, and cervix uteri.

The associated ectopic penis, scrotum, and penoscrotal transposition are the least common and probably the absolutely most unknown malformations of the male genitalia. The ectopic penis and scrotum are located in the perineum, whereas in the transposition the penis lies above the scrotum.

Keywords

Bladder exstrophy · Choristoma · Embryology . Cloaca $\cdot$ Penoscrotal transposition 


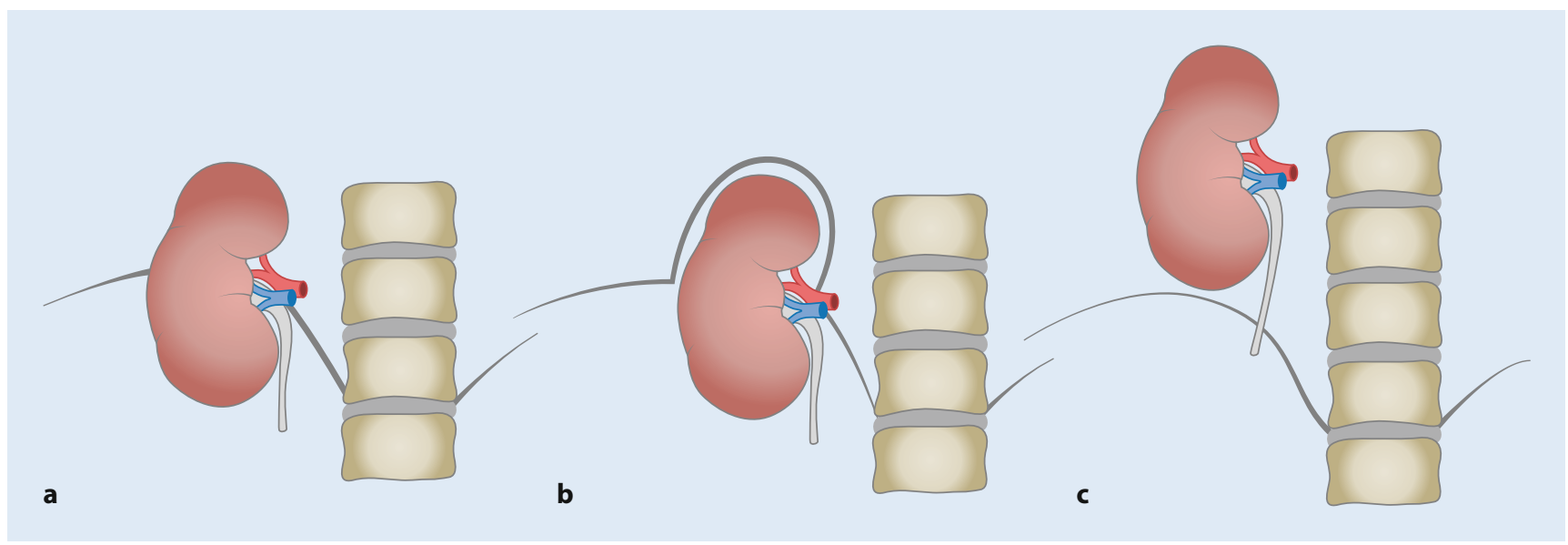

Abb. 2 ॥ Intrathorakal gelegene Nieren. a Subdiaphragmatisch, b Zwerchfelleventration, c intrathorakal. (Modifiziert nach Hill [19])

\begin{tabular}{ll|l}
\hline $\begin{array}{l}\text { Tab. } 1 \\
\begin{array}{l}\text { Frauen (Gesamtanteil 82,5 \%) } \\
\text { Anteil in \% }\end{array}\end{array}$ & Lokalisation & $\begin{array}{l}\text { Männer (Gesamtanteil 17,5 \%) } \\
\text { Anteil in \% }\end{array}$ \\
\hline 15 & Blasenhals & 10 \\
\hline 35 & Urethra & 60 \\
$<1$ & Rektum & $<1$ \\
\hline 20 & Vagina & - \\
\hline 25 & Vulva & - \\
\hline- & Samenblase & 20 \\
\hline- & Ductus deferens & 5 \\
\hline- & Utriculus prostaticus & 5 \\
\hline
\end{tabular}

im Bereich der Geschlechtsorgane liegen $[16,23,24]$. Die zuerst erwähnten Lokalisationen werden bei beiden Geschlechtern am häufigsten beobachtet. In den Geschlechtsorganen münden die ektopen Ureter bei den Frauen meist in der Vulva, bei den Männer in der Samenblase (Verteilung s. • Tab. 1).

Es soll nicht unerwähnt bleiben, dass die Ektopie bei über der Hälfte der Fälle von einer Verdoppelung des Ureters (Ureter duplex) begleitet wird [16, 23, 24], wobei grundsätzlich die Mündung des kranial gelegenen Ureters fehlgelagert ist.

\section{Klinik}

Die Inzidenz der Ureterektopie wird mit 1:1900 Personen geschätzt, könnte aber auch höher sein [6], weil manche Fälle symptomlos sind. Über $60 \%$ der Erkrankten sind Frauen [16, 23, 24]; in manchen Statistiken wird das Verhältnis männlich:weiblich sogar mit 1:10 angegeben [6].

Die Triade normale Blasenentleerung mit nachträglichem „Tröpfeln“ bei normaler Nierenfunktion ist ein klassisches Zeichen der Krankheit bei jungen Mädchen. Wenn das Orificium in der proximalen Urethra liegt, sind die Patienten kontinent. Vaginale Entleerungen täuschen eine Vaginitis oder Vulvitis vor [24]. Sonstige Komplikationen sind Pyelonephritis und Hydronephrose. Je weiter die Mündung des Ureters vom Trigonum entfernt ist, desto größer die Wahrscheinlichkeit, dass die betroffene Niere hypoplastisch ist [22].

Ektopien, bei denen ein einzelner Harnleiter vorliegt, sind gelegentlich mit anderen Missbildungen kombiniert, am häufigsten mit einer Analatresie [16], die allein oder im Rahmen eines VATERSyndroms (VATER, „vertebral defects, imperforate anus, tracheo-esophageal fistula, renal and radial dysplasia“) oder dessen Varianten auftritt [16, 37].

\section{Harnblasenekstrophie- Epispadie-Komplex und kloakale Ekstrophie}

Die Blasenekstrophie ist keine vollständige Ektopie, sondern ein fehlender Verschluss der vorderen Bauchwand, sodass die Blasenschleimhaut kontinuierlich in die umgebende Haut übergeht.

\section{Embryologie}

Die Kloake bildet das gesamte Ende des Hinterdarms und des Urogenitaltraktes. Sie ist von Endoderm ausgekleidet und die kloakale Membran liegt unmittelbar unter dem Ektoderm. Die Verdichtung des Mesenchyms zwischen diesen beiden Strukturen führt zur Bildung des Septum urorectale, das die Kloake in zwei Teile trennt. Aus dem dorsalen unteren Teil entstehen das Rektum und der Analkanal, aus dem ventralen oberen Teil entsteht der Sinus urogenitalis, aus dem sich die Harnblase und die Urethra sowie bei den Frauen noch der untere Teil der Vagina entwickeln. Wenn das Mesoderm nicht das Ektoderm vom Endoderm trennt, dann bleiben nach Ruptur der Kloakenmembran Urethra und Blasenplatte im unteren Abdomen unverschlossen zurück. Wenn dies nach Trennung von Urogenitalsinus und Enddarm geschieht, entsteht die Blasenekstrophie mit Epispadie, vor dieser Trennung hingegen eine kloakale Ekstrophie [4, 27]. 

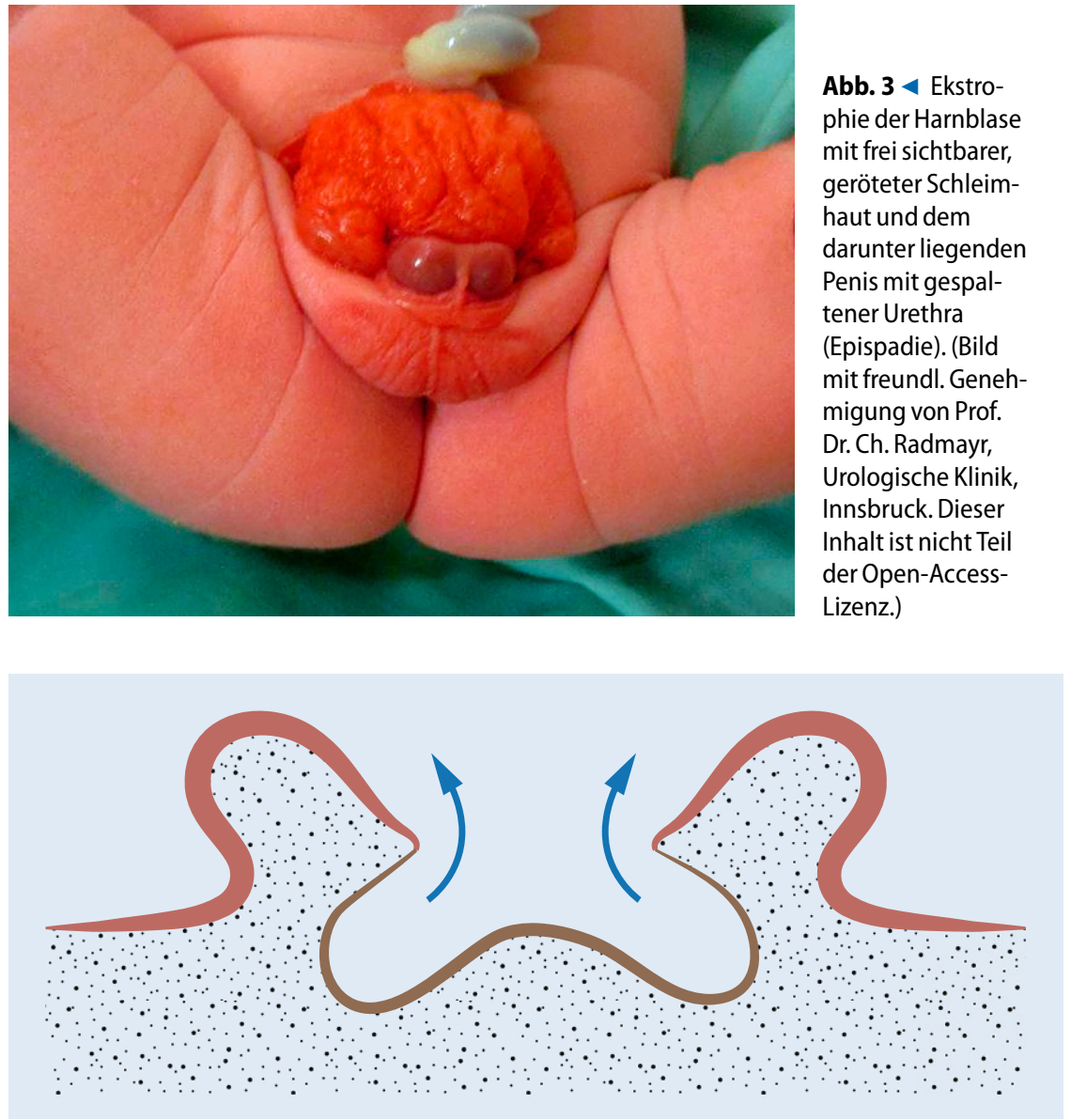

Abb. 4 ॥ Kloakale Ekstrophie. Die gespaltene Harnblase (rot) liegt beidseits des offenen Darmes (braun). (Modifiziert nach Hill [19])

\section{Morphologie}

Makroskopisch klafft in der Bauchwand eine Lücke, die von unterhalb des Nabels bis zur Symphyse reicht (• Abb. 3). Der Grund der Lücke besteht aus der Hinterwand der Harnblase, die wie eine rote, fleischige Masse aussieht. Die Orificia beider Ureteren, aus denen ständig Harn tröpfelt, sind frei sichtbar. Bei Knaben geht die Blase in die gespaltene Urethra über, die an der Oberfläche des abgeflachten und verkürzten Penis liegt und in einer gespaltenen Glans mündet. Bei Mädchen besteht außer der offenen Harnröhre eine gespaltene Klitoris, die Schamlippen sind gespreizt, der Eingang zur Vagina liegt direkt hinter der Urethrarinne.

Mikroskopisch geht das Urothel nahtlos in das Plattenepithel der Epidermis über. Die Blasenschleimhaut ist ödematös mit erweiterten Kapillaren, die
Abb. $3<$ Ekstrophie der Harnblase mit frei sichtbarer, geröteter Schleimhaut und dem darunter liegenden Penis mit gespaltener Urethra (Epispadie). (Bild mit freundl. Genehmigung von Prof. Dr.Ch. Radmayr, Urologische Klinik Innsbruck. Dieser Inhalt ist nicht Teil der Open-AccessLizenz.)

\section{Klinik}

Die Inzidenz der Erkrankung in den verschiedenen europäischen Ländern schwankt zwischen 0,6-4,7/100.000 Geburten. Das Mann-Frau-Verhältnis wird mit ungefähr 2:1 angegeben. Die höchste Rate (8/100.000) wird bei amerikanischen Indianern beobachtet [12]. Wegen der pränatalen Ultraschalldiagnose wird allerdings in einem Viertel der Fälle ein Schwangerschaftsabbruch durchgeführt [7].

Monozygote Zwillingen erkranken 7,5-mal häufiger als dizygote [33]. Nach Untersuchungen einiger Forschungsgruppen sei für diese familiäre Häufung das Empfänglichkeitsgen ISL1 verantwortlich, was in späteren Untersuchungen allerdings nicht bestätigt werden konnte [3]. Zahlreiche Studien belegen teratogene Effekte des Rauchens und Alkoholkonsums während der Schwangerschaft. Für gewisse potenzielle teratogene Medikamente (z.B. Diazepam, Heparin, Natriumvalproat, Phenobarbital, Phenytoin) gibt es nur Einzelbeobachtungen [12]. Fraglich ist auch, ob die Missbildung bei Schwangerschaften nach Invitro-Fertilisation tatsächlich häufiger als erwartet auftritt [38].

Kinder mit Ekstrophie leiden vermehrt an Anomalien des Gastrointestinal- und Genitaltraktes. Omphalozele (88-100\%) und Kurzdarmsyndrom (bis $46 \%)$ sind die häufigsten Begleiterkrankungen [12].

Die Blasenekstrophie ist ein urologischer Notfall - die „nackte“ Blasenschleimhaut muss sofort geschützt werden. Moderne Rekonstruktionsstrategien sehen einen Verschluss der Blase in den ersten $48 \mathrm{~h}$ nach der Geburt vor, danach wird stufenweise das Urogenitale rekonstruiert [12].

\section{Kloakale Ekstrophie}

Die ekstrophe Harnblase ist gespalten, zwischen den beiden Hälften liegt der ileozökale Anteil des Darms, der gegen das Ileum offen und gegen den Dickdarm (Hinterdarm) verschlossen ist (• Abb. 4). Das Rektum und der Anus fehlen (Agenesie).

\section{Hodenektopie}

Im Gegensatz zum kryptorchen Hoden liegt der ektope Hoden außerhalb der Deszensuswege. Eine abnorme Fixierung des Gubernaculum testis an die vordere Bauchwand während des transabdominalen Deszensus und/oder eine aberrante Migration während der inguinoskrotalen Phase werden als Ursache vermutet [31]. 


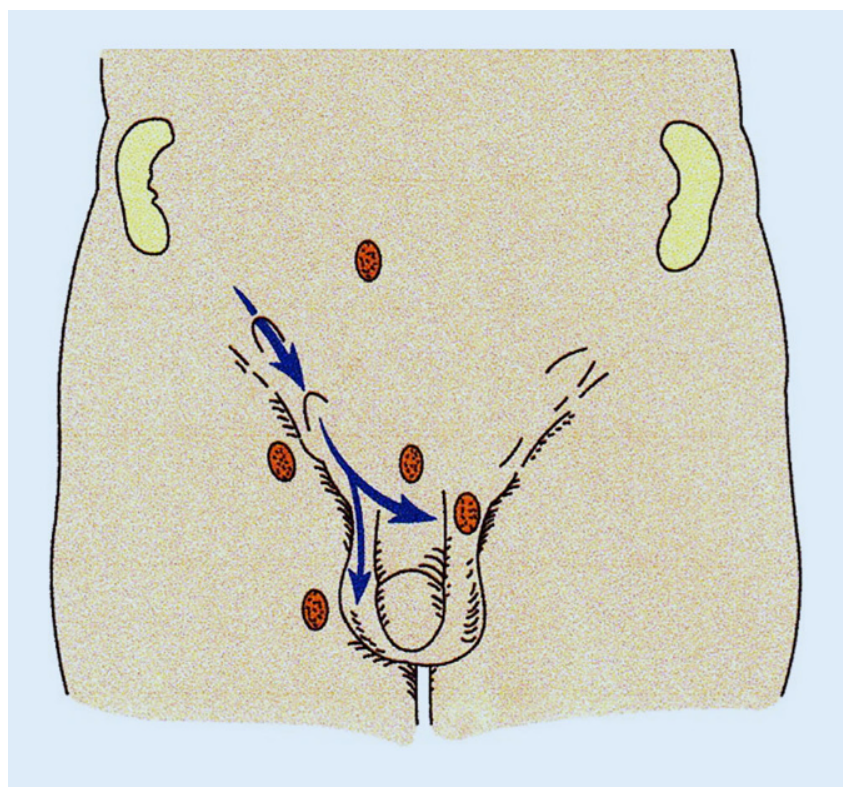

Abb. $5<$ Verschiedene mögliche Lagen des ektopen Hodens. Die blauen Pfeile zeigen den physiologischen Deszensusweg. (Aus Mikuz und Mazal [26], dieser Inhalt ist nicht Teil der OpenAccess-Lizenz.)
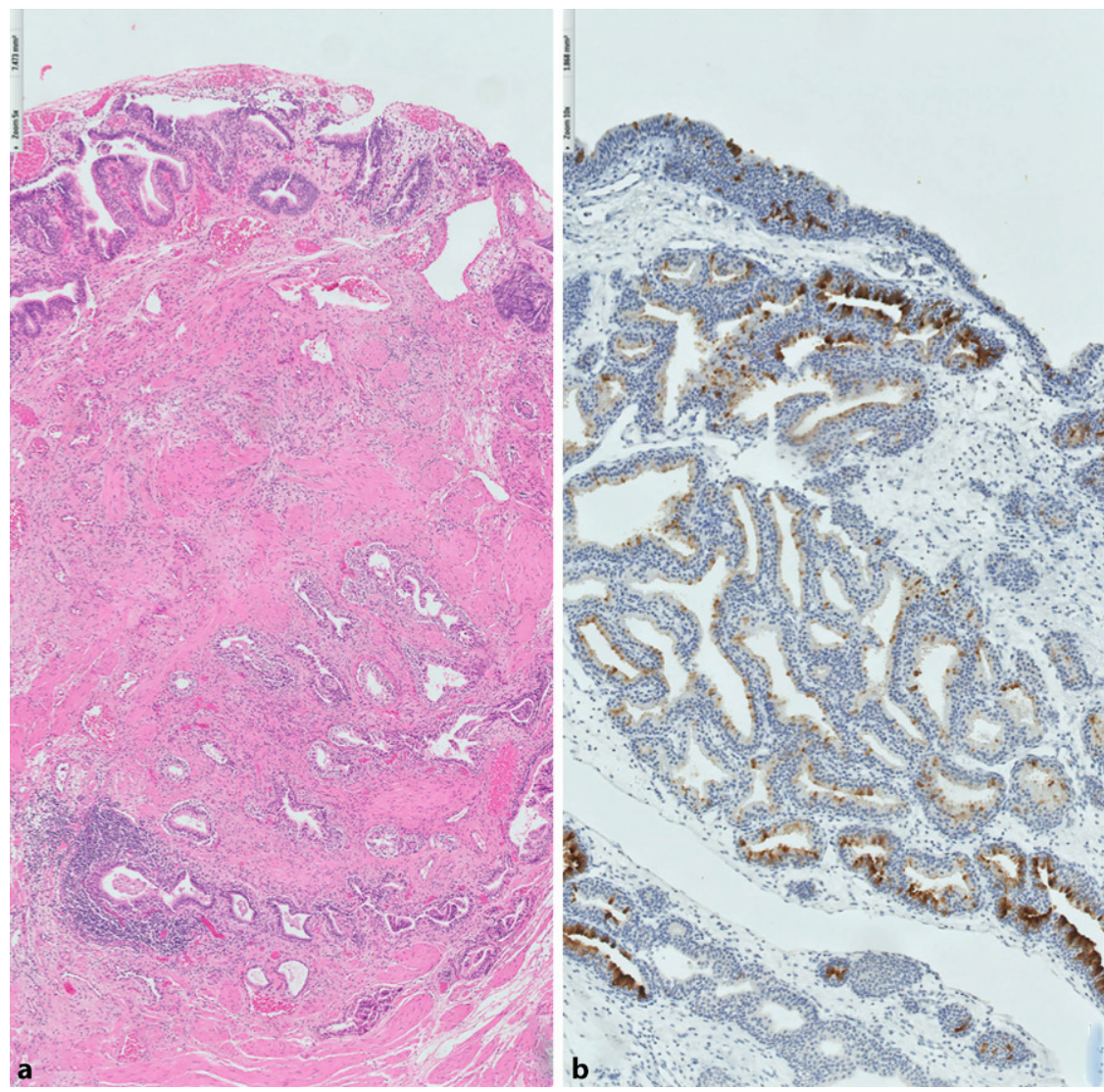

Abb. 6 \ Ektopie der Prostata. a Unter die Blasenschleimhaut verlagerte Prostatadrüsen. HE-Färbung, Vergr. 10:1. b Positiv mit PSA-Antikörper reagierende Prostataepithelien. Vergr. 10:1. (Bild mit freundl. Genehmigung von Prof. Dr. A. Lopez Beltran, Pathologie, Universität Cordoba. Dieser Inhalt ist nicht Teil der Open-Access-Lizenz.)

\section{Morphologie}

Entsprechend der abnormen Lage des Hodens unterscheidet man folgende Formen ([26]; • Abb. 5):

- Femorale Hodenektopie: Der Hoden liegt unter der Oberschenkelhaut.

- Perineale Hodenektopie: Der Hoden liegt im Bereich des Damms.

- Penile Hodenektopie: Der Hoden liegt im Bereich des Penisschafts.

- Transverse Hodenektopie: Verlagerung eines Hodens in das gegenseitige Skrotalfach.

- Tiefe abdominelle Hodenektopie: Der Hoden liegt präperitoneal im Bauchraum, oft zwischen den Fasern des M. obliquus internus.

Im Gegensatz zu den kryptorchen sind ektope Hoden zunächst normal groß und mikroskopisch normal strukturiert. Mit der Zeit kommt es aber zu temperaturbedingten Schädigungen und zur Atrophie des Parenchyms, wie dies beim Kryptorchismus der Fall ist.

Klinik

Schätzungsweise liegen $5 \%$ der vermeintlichen kryptorchen Hoden außerhalb der anatomischen Deszensuswege. Bisher wurden 271 Fälle veröffentlicht, wobei in den meisten Fällen die transverse Hodenektopie beschrieben wurde [31]. Diese ist nicht selten mit dem Müller-Gang-Persistenzsyndrom kombiniert [32]. Die ektope Lage wird in den meisten Fällen erst während einer chirurgischen Exploration festgestellt, die unter dem Verdacht eines Kryptorchismus bzw. einer Monorchie durchgeführt wird. Chirurgisch gelingt es nur selten, den Hoden in das Skrotum zu verlagern. Wegen des erhöhten Tumorrisikos [32] sollten atrophe Hoden entfernt werden.

\section{Prostataektopie}

Es handelt sich nicht um eine Fehllagerung des gesamten Organs, sondern um versprengte Prostatadrüsen, die vor allem in der Blasenwand ( $85 \%)$ und in der Urethra zu finden sind [17]. Einige Fälle wurden aber auch in Darmwand, Anus, im perikolischen Fettgewebe, in Milz, Sa- 


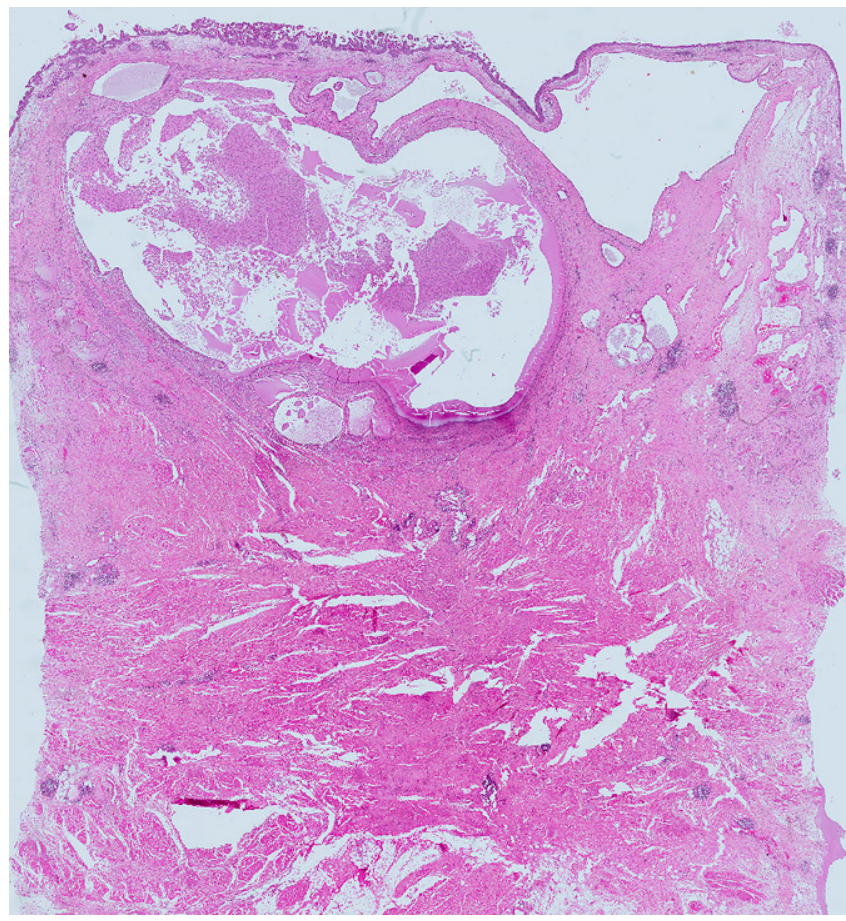

Abb. $7<$ Tumor vortäuschende, zystisch ausgeweitete Prostatadrüsen in der Blasenwand, die zu einer unnötigen Zystoprostatektomie führten. HEFärbung, Vergr. 1:1 (Bild und Anamnese mit freundl. Genehmigung von Prof. Dr. R. Montironi, Pathologie, Universität Ancona. Dieser Inhalt ist nicht Teil der Open-AccessLizenz.)

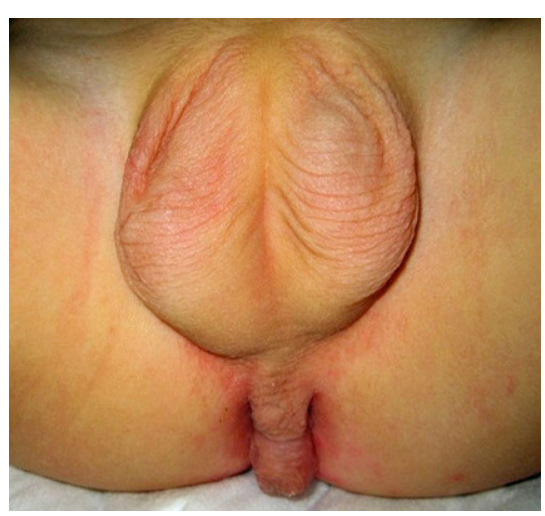

Abb. 9 A Transposition von Penis und Skrotum. (Aus Somoza et al. [36]; mit freundl. Genehmigung von Wolters Kluwer Medknow Publications. Dieser Inhalt ist nicht Teil der Open-AccessLizenz.)

menblase, Hoden und der Cervix uteri sowie Vagina beschrieben [14].

Die Entstehungsgeschichte dieser Läsion ist völlig unklar. Die Prostata entsteht aus der dorsalen Wand der Urethra durch Knospenbildung, die endodermalen Ursprungs ist. Das Stroma hingegen entsteht aus dem Mesoblast unter Dihydrotestosteroneinfluss. Ob die Prostatadrüsen durch Wandern in eine ektope Lage kommen oder aber es sich um eine Metaplasie des jeweiligen ortsständigen Drüsengewebes handelt, wird in allen diesbezüglichen Publikationen offen gelassen [14, 17]. Auch bei Prostata feminina oder Skene-Drüsen, die bei der Frau paraurethral liegen, weiß man nicht, ob sie embryonale Reste oder eine Metaplasie der Zervixdrüsen darstellen [17].

\section{Morphologie}

Im Bereich der Harnblase und der Urethra ist die Ektopie flach unter der normalen Schleimhaut ausgebreitet, kann aber auch papillär exophytisch wachsen (๑Abb.6). Beide Wachstumsformen bestehen aus typischen Prostatadrüsen, bei denen man bereits in der HE-Färbung sekretorische und basale Zellen deutlich erkennt. In knapp der Hälfte der Fälle findet man in der Lichtung auch Corpora amylacea. Die darüberliegende Schleimhaut zeigt oft entzündliche Veränderungen. Immunhistochemisch sind die Zellen PSA- und PSAP-positiv (• Abb.6), allerdings reagieren mit diesen Antikörpern bei beiden Geschlechtern auch die Epithelien der periurethralen Drüsen positiv. Deswegen wird die Verwendung von P501S empfohlen, weil dieser Antikörper sehr spezifisch gut- und bösartige Prostataepithelien färbt. Basale und sekretorische Zellen der normalen und hyperplastischen, aber nicht karzinomatösen Prostatadrüsen sind auch CD10positiv. Der Antikörper ist ein guter

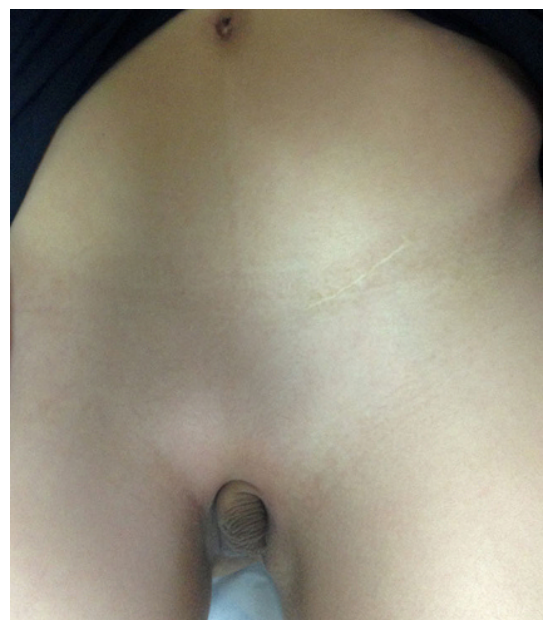

Abb. $8 \Delta$ Kombinierte perineale Ektopie von Penis und Skrotum. (Aus Sobral et al. [35], @ Prof. Dr. E. Damázio et al. CC-BY 4.0)

Marker für Gewebe mesonephrischer Abstammung [17].

Fulton et al. [14] vertreten die Meinung, dass die beschriebenen Fälle von Zystadenomen der Prostata, die entweder kaum in Verbindung mit der Drüse selbst oder deutlich getrennt davon lagen, im ektopen Gewebe entstanden sind. Im ektopen Prostatagewebe wurden auch organtypische Adenokarzinome beschrieben [15].

\section{Klinik}

Die Behauptung [17], die Ektopie der Prostata sei in der spezifischen Prostataliteratur ",underreported“ ist durchweg berechtigt, weil die Läsion in den meisten Schriften gar nicht oder nur mit einem kurzen Hinweis erwähnt wird. Dies ist insofern unverständlich, weil die Läsion im Gegensatz z. B. zur einfachen Nierenektopie fast immer klinische Symptome verursacht. Bei Lokalisation im unteren Harntrakt sind Hämaturie, Dysurie und Nykturie die häufigsten Symptome. Die etwas selteneren Obstruktionserscheinungen sind tumorverdächtig und ziehen chirurgische Maßnahmen bis zur unnötigen radikalen Zystoprostatektomie nach sich (•Abb. 7). Besonders zystadenomähnliche Ektopien auBerhalb der Prostata machen sich auch durch Verdrängung oder Darmobstruktion bemerkbar. In solchen Fällen gibt es für Pathologen differenzialdiagnosti- 
sche Schwierigkeiten, wenn man an die Prostataektopie nicht denkt.

\section{Penis-/Skrotumektopie und penoskrotale Transposition}

Mit etwa 63 beschriebenen Fällen [13] handelt es sich um die seltensten und wahrscheinlich die unbekanntesten Missbildungen der männlichen Geschlechtsorgane überhaupt. Die seltenste Form (20 Fälle) ist die gemeinsame Verlagerung vom Penis und Skrotum in das Perineum ([35]; - Abb. 8). Bei der Transposition liegt hingegen der Penis unter dem Skrotum (• Abb. 9) oder zwischen beiden Hoden in einer Furche des gespaltenen Hodensacks [13, 36]. Die Missbildung ist oft nicht nur mit anderen Anomalien des Genitaltraktes, sondern auch mit solchen von Gastrointestinal- und Herz-Kreislauf-Organen sowie des zentralen Nervensystems vergesellschaftet [12]. $60 \%$ der Patienten sind geistig retardiert. Die Kombination mit dem Klinefelter-Syndrom wurde ebenfalls einige Male beschrieben [20]. Diese Ektopien können chirurgisch zufriedenstellend korrigiert werden [35, 36].

Der embryologische Mechanismus dieser Ektopie bleibt unklar. Normalerweise wandert die Labioskrotalschwellung zwischen der 9. und 11. Schwangerschaftswoche kaudal und medial und fusioniert dann in der 12. Woche hinter dem Penis. Später deszendieren die Hoden aus dem Bauch in den Sack herab. Eine abnorme Lage des Genitalhöckers oder die abnorme Migration bzw. eine zu frühe Spaltung der Labioskrotalschwellung werden als mögliche Ursachen dieser Ektopie diskutiert [13].

\section{Fazit für die Praxis}

Die Diagnostik der Ektopien der Nieren, der ableitenden Harnwege, der Blasenekstrophie und der extrem seltenen Ektopien der äußeren männlichen Geschlechtsorgane ist eine Domäne der Klinik. Trotzdem gehört das Grundwissen über diese Krankheiten zu unserem Fach und kann schon aus didaktischen Gründen nicht einfach ignoriert werden.
Ganz anders verhält sich die Ektopie der Prostatadrüsen. Die Unkenntnis dieser Läsion kann zu einer pathohistologischen Fehldiagnose führen, die für den Patienten katastrophale Folgen haben kann. Die Erscheinung von Drüsen in Blasenschleimhautbiopsien des Mannes oder von "Zystadenomen" im kleinen Becken sollte deswegen mithilfe der Immunhistochemie gründlich abgeklärt werden.

\section{Korrespondenzadresse}

\section{Prof. Dr. G. Mikuz}

Institut für Pathologie, Universität Innsbruck Müllerstraße 44, 6020 Innsbruck, Österreich Gregor.Mikuz@i-med.ac.at

Funding. Open access funding provided by University of Innsbruck and Medical University of Innsbruck.

\section{Einhaltung ethischer Richtlinien}

Interessenkonflikt. G. Mikuz gibt an, dass kein Interessenkonflikt besteht.

Dieser Beitrag beinhaltet keine von den Autoren durchgeführten Studien an Menschen oder Tieren.

Open Access. Dieser Artikel wird unter der Creative Commons Namensnennung 4.0 International Lizenz (http://creativecommons.org/licenses/by/4.0/deed. de) veröffentlicht, welche die Nutzung, Vervielfältigung, Bearbeitung, Verbreitung und Wiedergabe in jeglichem Medium und Format erlaubt, sofern Sie den/die ursprünglichen Autor(en) und die Quelle ordnungsgemäßnennen, einen Linkzur Creative Commons Lizenz beifügen und angeben, ob Änderungen vorgenommen wurden.

\section{Literatur}

1. Abeshouse BS, Bhitsitkul I (1959) Crossed renal ectopia with and without fusion. Urol Int 9:63-91

2. Aliotta PJ, Seidel FG, Karp M, Greenfield SP (1987) Renal malposition in patients with omphalocele. JUrol 137:942-944

3. Arkani S, Cao J, Lundin J, Nilsson D et al (2018) Evaluation of the ISL1 gene in the pathogenesis of bladder exstrophy in a Swedish cohort. Hum Genome Var 5:18009

4. Austin PF, Homsy YL, Gearhart JP et al (1998) Prenatal diagnosis of cloacal exstrophy. J Urol 160:1179-1181

5. Babu V, Skandhan AKP et al (2018) Ectopic kidney https://radiopaedia.org/articles/ectopic-kidney1.Zugegriffen: 12.08.2018

6. Bell DJ, Abdrabou A et al (2018) Ectopic ureter. https://radiopaedia.org/articles/ectopic-ureter. Zugegriffen: 12.08.2018

7. Cromie WJ, Lee K, Houde K, Holmes L (2001) Implications of prenatal ultrasound screening in the incidence of major genitourinary malformations. JUrol 165:1677-1680
8. Culp DA (1964) The histology oft he extrophied bladder. JUrol 91:538-548

9. Dell'Atti L, Galosi AB (2017) Right ectopic intrathoracic kidney: Unusual clinical presentation in a young patient affected by scrotal varicocele. Arch Ital Urol Androl 89:323-324

10. Delson B (1975) Ectopic kidney in obstetrics and gynecology. NYState J Med 75:2522-2526

11. Dretler SP, Olsson C, Pfister RC (1971) The anatomic, radiologic and clinical characteristics of the pelvic kidney: an analysis of 86 cases. J Urol 105:623-627

12. Ebert AK, Reutter H, Ludwig M, Rösch WH (2009) The exstrophy-epispadias complex. Orphanet J Rare Dis 4:23

13. Fahmy MA, El Shennawy AA, Edress AM (2014) Spectrum of penoscrotal positional anomalies in children. Int J Surg 12:983-988

14. Fulton RS, Rouse RV, Ranheim EA (2001) Ectopic prostate: case report of a presacral mass presenting with obstructive symptoms. Arch Pathol Lab Med 125:286-288

15. Gardner JM, Khurana H, Leach FS, Ayala AG (2010) Adenocarcinoma in ectopic prostatic tissue at dome of bladder: a case report of a patient with urothelial carcinoma of the bladder and adenocarcinoma of the prostate. Arch Pathol Lab Med 134:1271-1275

16. Gill B (1980) Ureteric ectopy in children. Br J Urol 52:257-263

17. Halat S, Eble JN, Grignon DJ, Lacy $S$ et al (2011) Ectopic prostatic tissue: histogenesis and histopathological characteristics. Histopathology 58:750-758

18. Hertz M, Rubinstein ZJ, Shahin N, Melzer M (1977) Crossed renal ectopia: clinical and radiological findings in 22 cases. Clin Radiol 28:339-344

19. Hill GS (1989) Renal and ureteral anomalies. In: Hill GS (Hrsg) Uropathology, Bd. I. Churchill Livingstone, New York Edinburgh London Melbourne, S $1-80$

20. Hodhod A, Umurangwa F, El-Sherbiny M (2015) Prepubertal diagnosis of Klinefelter syndrome due to penoscrotal malformations: Case report and review of literature. Can Urol Assoc J 9:E333-E336

21. Little MH, Brennan J, Georgas Ket al (2007) A highresolution anatomical ontology of the developing murine genitourinary tract. Gene Expr Patterns 7:680-699

22. Mackie GG, Stephens FD (1977) Duplex kidneys: a correlation of renal dysplasia with position of the ureteric orifice. Birth Defects Orig Artic Ser 13:313-321

23. Mandell J, Bauer SB, Colodny AH et al (1981) Ureteral ectopia in infants and children. J Urol 126:219-222

24. Mangin P, Cukier J (1982) Abouchement ectopiques extravesicaux de I' uretere:problemes diagnostiquesm. Nouv Press Med 11:1135-1138

25. McDonald JH, McClellan DS (1957) Crossed renal ectopia. Am J Surg 93:995-1002

26. Mikuz G, Mazal P (2016) Hoden und Infertilität beim Mann. In: Amann K, Kain R, Klöppel G (Hrsg) Pathologie. Urogenitale und Endokrine Organe,Gelenke und Skelett. Springer, Berlin Heidelberg

27. Muecke EC (1964) The role of the cloacal membrane in exstrophy: the first successful experimental study. JUrol 92:659-667

28. Musarrat H, Erum S, Amir B, Beenish K (2010) Diagnosis of unilateral thoracic kidney by color dopplerultrasound. J Med Ultrasound 18:34-38

29. Natarajan G, Jeyachandran D, Subramaniyan B etal (2013) Congenital anomalies of kidney and hand: a review. Clin Kidney J 6:144-149 
30. Pinckney LE, Moskowitz PS, Lebowitz RL, Fritsche P (1978) Renal malposition associated with omphalocele. Radiology 129:677-682

31. Punwani VV, Wong JSY, Lai CYH et al (2017) Testicular ectopia: Why does it happen and what do we do? JPediatr Surg 52:1842-1847

32. Raj V, Redkar R, Krishna S, Tewari S (2017) Rare case of transverse testicular ectopia-Case report and review of literature. Int J Surg Case Rep 41:407-410

33. Reutter H, Shapiro E, Gruen JR (2003) Seven new cases of familial isolated bladder exstrophy and epispadias complex (BEEC) and review of the literature. Am J Med Genet A 120A:215-221

34. Singh J, Singh N, Kapoor K, Sharma M (2015) Bilateral malrotation and a ongenital pelvic kidney with varied vasculature and altered hilar anatomy. Case Rep Med 2015:ID 848949

35. Sobral Filho DSR, Silva HDD, Damázio E (2017) Surgical correction of ectopic penis and scrotum associated with bilateral orchidopexy. Einstein (Sao Paulo) 15:223-225

36. Somoza I, Palacios MG, Mendez R, Vela D (2012) Complete penoscrotal transposition: A three-stage procedure. Indian JUrol 28:450-452

37. Sotolongo JR Jr, Rose J, Strauss L, Gribetz M (1982) Single vaginal ectopic ureter and the VATER syndrome.J Jrol 127:1181-1182

38. Wood HM, Trock BJ, Gearhart JP (2003) In vitro fertilization and the cloacal-bladder exstrophyepispadias complex: is there an association? J Urol 169:1512-1515

\section{Erratum}

Pathologe 2018 $39: 423$

https://doi.org/10.1007/s00292-018-0479-x

Online publiziert: 13. August 2018

C) Springer Medizin Verlag GmbH, ein Teil von Springer Nature 2018

CrossMark

\section{Schüttler ${ }^{1}$ N. Buschhüter ${ }^{2} \cdot$ C. Döllinger ${ }^{3} \cdot$ L. Ebert $^{4} \cdot$ M. Hummel ${ }^{5} \cdot$ J. Linde $^{6}$.} H.-U. Prokosch ${ }^{1} \cdot$ R. Proynova ${ }^{4} \cdot$ M. Lablans ${ }^{4}$

'Lehrstuhl für Medizinische Informatik, Friedrich-Alexander-Universität Erlangen-Nürnberg, ErlangenTennenlohe, Deutschland

${ }^{2}$ Zentralisierte Biomaterialbank der Medizinischen Fakultät der RWTH Aachen University, Aachen Deutschland

${ }^{3}$ BioMaterialBank Heidelberg, Universitätsklinikum Heidelberg, Heidelberg, Deutschland

${ }^{4}$ Medizinische Informatik in der Translationalen Onkologie, Deutsches Krebsforschungszentrum, Heidelberg, Deutschland

${ }^{5}$ Zentrale Biomaterialbank (ZeBanC) und German Biobank Node (GBN), Charité-Universitätsmedizin Berlin, Berlin, Deutschland

${ }^{6}$ UMG Biobank, Universitätsmedizin Göttingen, Göttingen, Deutschland

\section{Erratum zu: Anforderungen an eine standortübergreifende Biobanken-IT-Infrastruktur}

\section{Erhebung des Stakeholderinputs zum Aufbau eines Biobankennetzwerks der German Biobank Alliance (GBA)}

\author{
Erratum zu: \\ Pathologe (2018) \\ https://doi.org/10.1007/s00292-018- \\ 0435-9
}

Bei der Publikation „Anforderungen an eine standortübergreifende BiobankenIT-Infrastruktur. Erhebung des Stakeholderinputs zum Aufbau eines Biobankennetzwerks der German Biobank Alliance (GBA)“ handelt es sich um einen Teil der Dissertation von Frau Christina Schüttler. Die Arbeit dient der Erfüllung von Voraussetzungen zur Erlangung des Doktorgrades Dr. rer. biol. hum. an der Friedrich-AlexanderUniversität Erlangen-Nürnberg.

\section{Korrespondenzadresse}

\section{Schüttler}

Lehrstuhl für Medizinische Informatik, Friedrich-Alexander-Universität ErlangenNürnberg

Wetterkreuz 13, 91058 Erlangen-Tennenlohe, Deutschland

christina.schuettler@fau.de

Die Online-Version des Originalartikels ist unter https://doi.org/10.1007/s00292-018-0435-9zu finden. 